\title{
Comparing Real-Time Self-Tracking and Device-Recorded Exercise Data in Subjects with Type 1 Diabetes
}

\author{
Danielle Groat ${ }^{1,2}$ Hyo Jung Kwon ${ }^{2}$ Maria Adela Grando 2,3 Curtiss B. Cook ${ }^{2,3}$ Bithika Thompson ${ }^{3}$
}

${ }^{1}$ Department of Biomedical Informatics, University of Utah, Salt Lake
City, Utah, United States
${ }^{2}$ Department of Biomedical Informatics, Arizona State University,
Scottsdale, Arizona, United States
${ }^{3}$ Division of Endocrinology, Mayo Clinic Arizona, Scottsdale, Arizona,
United States

Appl Clin Inform 2018;9:919-926.

\author{
Address for correspondence Danielle Groat, PhD, 421 Wakara Way \\ Suite 140, Salt Lake City, UT 84108, United States \\ (e-mail: danielle.groat@utah.edu).
}

\section{Abstract}

Background Insulin therapy, medical nutrition therapy, and physical activity are required for the treatment of type 1 diabetes (T1D). There is a lack of studies in real-life environments that characterize patient-reported data from logs, activity trackers, and medical devices (e.g., glucose sensors) in the context of exercise.

Objective The objective of this study was to compare data from continuous glucose monitor (CGM), wristband heart rate monitor (WHRM), and self-tracking with a smartphone application (app), iDECIDE, with regards to exercise behaviors and rate of change in glucose levels.

Methods Participants with T1D on insulin pump therapy tracked exercise for 1 month with the smartphone app while WHRM and CGM recorded data in real time. Exercise behaviors tracked with the app were compared against WHRM. The rate of change in glucose levels, as recorded by CGM, resulting from exercise was compared between exercise events documented with the app and recorded by the WHRM.

Results Twelve participants generated 277 exercise events. Tracking with the app aligned well with WHRM with respect to frequency, 3.0 (2.1) and 2.5 (1.8) days per week, respectively $(p=0.60)$. Duration had very high agreement, the mean duration from the app was 65.6 (55.2) and 64.8 (54.9) minutes from WHRM $(p=0.45)$. Intensity had a low concordance between the data sources (Cohen's kappa $=0.2$ ). The mean rate of change of glucose during exercise was $-0.27 \mathrm{mg} /\left(\mathrm{dL}^{*} \mathrm{~min}\right)$ and was not significantly different between data sources or intensity $(p=0.21)$.

Conclusion We collated and analyzed data from three heterogeneous sources from free-living participants. Patients' perceived intensity of exercise can serve as a surrogate for exercise tracked by a WHRM when considering the glycemic impact of exercise on self-care regimens.

\section{Background and Significance}

Type 1 diabetes (T1D) is a complex, chronic disease which requires patients to engage in certain self-management strategies to avoid acute and long-term complications. ${ }^{1-3}$ For instance, adherence to insulin therapy, medical nutrition therapy, and physical activity are the ways and means by which individuals with T1D achieve the objective of optimizing glycemic control. ${ }^{4-6}$ Patients are more likely to adhere to selfmanagement regimens that incorporate personal lifestyle received

July 6, 2018

accepted after revision

October 27, 2018 (c) 2018 Georg Thieme Verlag KG Stuttgart · New York
DOI https://doi.org/ 10.1055/s-0038-1676458. ISSN 1869-0327. 
preferences. ${ }^{7-9}$ However, incorporating physical activity can be challenging for individuals with T1D due to the multiple factors that influence glucose levels, such as the duration and intensity of exercise, the timing and quantity of delivered insulin, and carbohydrates consumed. Recommendations for compensating for exercise include disconnecting from the pump, adjusting bolus and/or basal insulin, consuming carbohydrates, and waiting for glucose levels to be at an appropriate concentration. ${ }^{6,10}$ It is also suggested that the current blood glucose concentration and the duration and intensity of the planned exercise should be considered before engaging in exercise to employ the appropriate compensation techniques to offset the acute effects of physical activity. ${ }^{6}$ Studies have found that most individuals with T1D resort to trial and error as they compensate for exercise, which can lead to considerable variability in self-care behaviors between and within participants. ${ }^{11,12}$

Ideally, clinicians would be able to review patient-generated exercise logs and compensation techniques employed, along with glucose data, to provide personalized recommendations aimed at improving glycemic control. However, one of the challenges in individualizing recommendations for exercise is the difficult nature in establishing the individual impact of physical activity on glucose levels in real-world, noncontrolled environments. The rate of change of glucose levels across time in minutes $(\Delta \mathrm{G} / \mathrm{min})$, is an indicator of how aggressively one should compensate for exercise, and has been found to be related to the intensity of physical activity, with moderate exercise decreasing glucose levels at a faster rate when compared with light exercise, and vigorous exercise sometimes increasing or decreasing glucose levels. ${ }^{5,6,10} \mathrm{Com}-$ monly used techniques for measuring physical activity include device-recorded data (e.g., pedometers or heart rate monitors), or self-reported information. The accuracy of self-reported exercise data has mixed levels of validity, while devices may fail to produce accurate measurements. ${ }^{13-15}$ Wearable activity monitors have been found to have good levels of reliability with respect to step counts and distance, with other measurements such as energy expenditure and sleep quality having lower validity. ${ }^{16}$ While one research group used a wrist-worn activity monitor to validate self-reported exercise in heart failure patients prescribed a walking routine, ${ }^{17}$ due to the shortcomings of these methods, there is no established standard for measuring exercise outside of controlled research settings. ${ }^{18-20}$ Although it has been suggested that combining methods to measure exercise in real-life outpatient settings may be the best way to accurately assess periods of exercise, this approach remains understudied. ${ }^{19,21}$

Studies have found that one-quarter to one-third of patients with T1D use apps to provide assistance with carb counting, recording glucose levels, and tracking exercise, with insulin pump users more likely to use apps than those on multiple daily injections. ${ }^{22-24}$ The authors have previously published data on a noncommercial smartphone application (app) called iDECIDE. ${ }^{25}$ To the best of our knowledge, iDECIDE is the first app that allows users to track the techniques they employ to compensate for meals, alcohol, and exercise, as illustrated in - Fig. 1. Additional functionalities of the app include self- tracking exercise duration, intensity, and start time. Outside of the scope of this study are features for self-tracking carbohydrates for meals and alcohol, assisting with carbohydrate counting, and providing insulin bolus recommendations. Utilization of the app in previous studies on T1D patients on insulin pumps has identified unexpected self-management behaviors related to how patients compensate for variables such as exercise. For instance, subjects reported on a survey that they adjusted the basal rate when exercising, but they recorded with the app that they instead took snacks before exercising. ${ }^{12}$ The app, which allows users to self-track diabetes management in real time, as opposed to tracking with paperbased logs, has not been compared with objective measures of exercise frequency, duration, and intensity.

\section{Objective}

Clinicians that treat patients with T1D grapple with interpreting patient-generated data from various sources, for example, carbohydrate and exercise logs (paper-based and/ or digital), activity trackers, glucose meters, and continuous glucose monitor (CGM), when addressing glycemic control. The objective of this study was to compile and compare exercise behaviors from free-living participants with T1D from three heterogeneous data sources found in outpatient settings to answer two questions: (1) how does an individual's exercise behaviors self-tracked via the app compare with real-time recording by a wristband heart rate monitor (WHRM)?, and (2) how does $\Delta \mathrm{G} / \mathrm{min}$, attributable to exercise tracked with the app, compare with $\Delta \mathrm{G} / \mathrm{min}$ attributable to exercise recorded by a WHRM?

\section{Methods}

\section{Participant Recruitment}

After obtaining Institutional Review Board approval, we recruited patients from an outpatient academic endocrinology clinic who were 18 to 70 years old and who were being treated for T1D. All participants used a Medtronic (Minneapolis, Minnesota, United States) insulin pump paired with CGM, and all owned a smartphone. Patients in fragile health, limited life expectancy, a history of mental health problems, advanced vascular disease or microvascular complications, and known history of severe hypoglycemia were excluded. Participants were required to have been receiving care in the clinic for at least 1 year. Patients were identified by facility researchers during routine office visits after which a recruitment appointment was set where the participants gave informed consent.

\section{Exercise Data}

At the recruitment appointment, participants were instructed to maintain their normal routines for 1 month. They were asked to log their exercise activities by using the self-tracking module of the app, which was installed on each participant's smartphone. Participants tracked exercise by recording perceived intensity as indicated by the "talk test" as: light (able to talk and sing), moderate (able to talk but not 

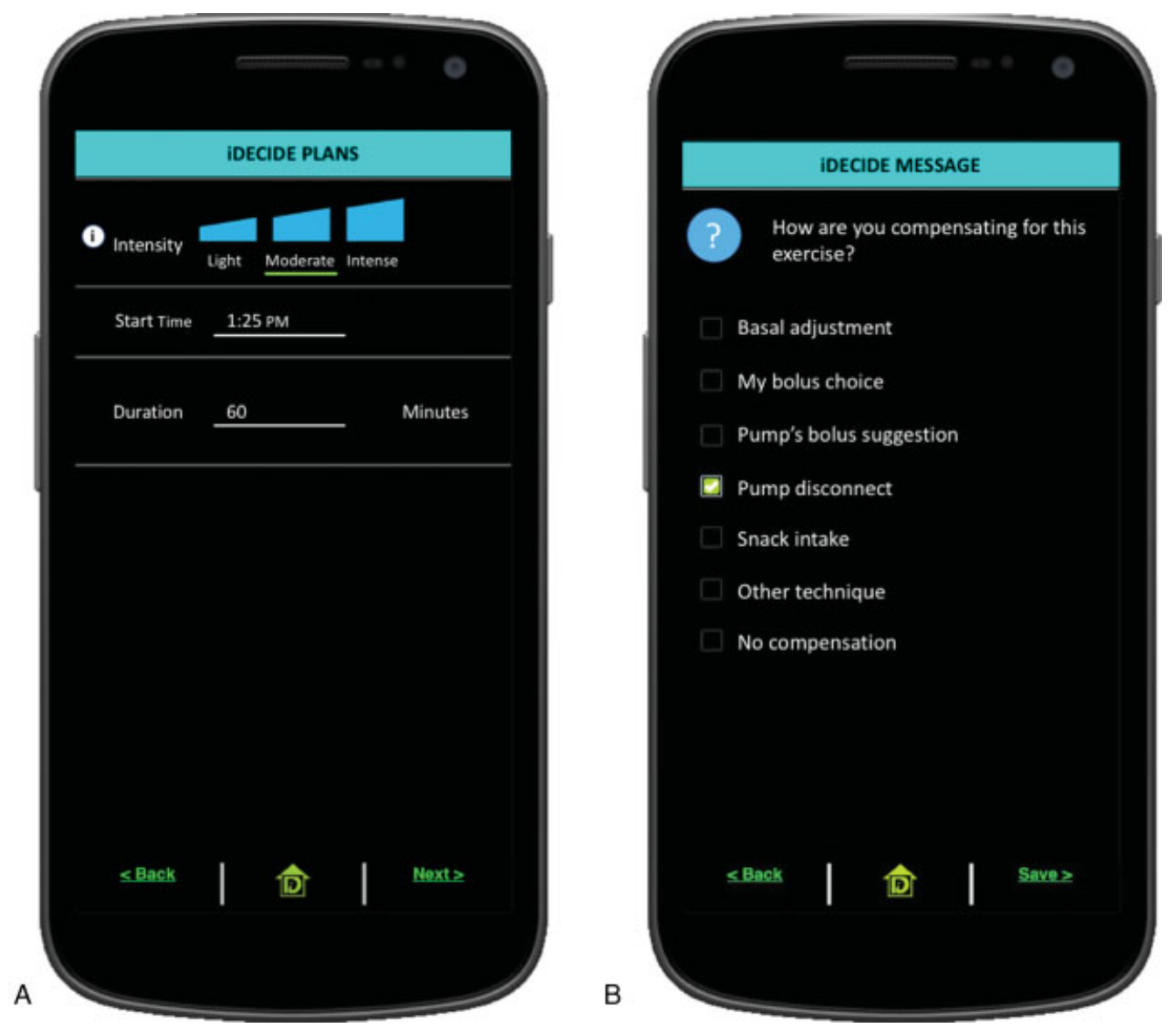

Fig. 1 Screenshots of the exercise self-tracking module of the smartphone app, iDECIDE. Participants logged exercise activity by (A) defining the start time, duration, and intensity, and (B) indicating the compensation techniques used.

sing), or vigorous (not able to talk). ${ }^{26}$ The talk test has been found to be a valid and reliable tool for monitoring exercise intensity across individuals of various health status. ${ }^{27}$ Additionally, exercise duration and start time were tracked ( - Fig. 1). At the end of the month, logs were downloaded in tabular format from the cloud-based Web service.

Simultaneously with the use of the app, an off-the-shelf WHRM which did not require any additional steps for calibration (FitBit Charge HR, San Francisco, California, United States) was distributed to each participant and placement was encouraged on the nondominant hand. The WHRM recorded heart rate in beats per minute, and start time and duration of exercise, accurate to the minute. During exercise events, a heart rate reading was displayed every 30 seconds, and an overall average heart rate for the event was also provided by the device manufacture's Web portal, otherwise a heart rate reading was provided every 5 minutes. The WHRM recorded exercise in real-time fashion for the 1month study period and was synchronized to the manufacturer's companion app for data uploads to the Web portal which granted researchers access to the data. ${ }^{28}$ While participants could directly initiate the reporting of start and finish times with the WHRM, the device was able to automatically detect exercise events and it recorded physical activity that was 15 minutes or longer. WHRM data were retrieved from the Web portal and were coded as tabular data.

\section{Glucose Readings}

Insulin pumps are capable of synchronizing and storing data from blood glucose meters and CGM. CGM delivers an interstitial glucose measurement to the insulin pump every 5 minutes. Participants were instructed to engage in selfcare as usual during the study, and no additional instructions were given regarding the calibration of the CGM sensor. Participants uploaded their insulin pump and CGM data to the manufacturer's Web portal and 1 month of corresponding data were downloaded in raw tabular format. Readings from the CGM were used to calculate $\Delta \mathrm{G} / \mathrm{min}$.

\section{Data Analysis}

\section{Comparison of Exercise Behaviors between the App and WHRM}

Exercise events self-tracked with the app and recorded by the WHRM were categorized as occurring in both data sources (i.e., matched), or as being present in only one data source (i.e., unmatched). Exercise events were considered to occur in both data sets if portions of the exercise event from both data sources occurred within a 1-hour widow. Exercise parameters (frequency, duration, intensity) from the app and the WHRM were compared. The average number of days per week participants engaged in exercise was determined for both the app and the WHRM. Exercise duration was also 
compared for exercise events that matched in both sources of data.

The intensity of exercise tracked by the participants with the app (light, moderate, or vigorous) was compared against the WHRM data. Although the heart rates from the WHRM were categorized into three intensities according to the manufacturer's proprietary algorithms, we categorized the average and highest heart rate recorded by the WHRM based on the formula: intensity $=$ heart rate $/(208-0.7 \times$ age $) .{ }^{26,29}$ The intensity was categorized as vigorous exercise for values between 0.7 and 0.85 , moderate from 0.5 to 0.7 , and light from 0.3 to 0.5 . The calculations were performed with age tracked in years, and heart rate in beats per minute.

\section{Comparison of $\Delta \mathrm{G} / \mathrm{min}$ between the App and WHRM}

A start and finish glucose reading were automatically extracted from the insulin pump data for exercise events from the app and the WHRM. Exercise events were included only if both a start and finish glucose value from the CGM within 10 minutes of the documented start and finish time for exercise were available. The $\Delta \mathrm{G} / \mathrm{min}$ was calculated as (finish glucose - start glucose) / exercise duration, where finish glucose and start glucose values were reported in $\mathrm{mg} / \mathrm{dL}$ and exercise duration was in minutes. The $\Delta \mathrm{G} / \mathrm{min}$ for exercise tracked with the app was compared with the $\Delta \mathrm{G} / \mathrm{min}$ for exercise documented by the WHRM.

\section{Statistical Analysis}

Cohen's kappa was used for categorical comparisons and twosided $t$-tests of unequal variance were used for numerical data, with pairing used for matched exercise events. Single-factor analysis of variance was used to compare $\Delta \mathrm{G} / \mathrm{min}$ categorized by intensity between the app and the WRHM. All results are reported as mean (standard deviation).

\section{Results}

\section{Participant Characteristics}

Twelve patients with T1D were recruited, all were Caucasian. All of the eight females and one male engaged in exercise and contributed complete data. One male participant did not engage in physical activity and was removed from any further analysis. Another male participant did not track any exercise with the app, but the WHRM recorded exercise events, while another male participant tracked exercise with the app, but was unable to upload exercise data from the WHRM to the manufacturer's companion portal. The average age of the 11 participants that generated exercise data was 48 (13.4) years and average length of T1D diagnosis was 29 (12.5) years. One participant used the Paradigm Revel 723 insulin pump paired with a Dexcom CGM, and all others used the MiniMed 530G pump paired with the Enlite CGM. Participants had an average of 13 (6.1) years of insulin pump therapy and average hemoglobin A1c (HbA1c) of $7.7 \%$ (1.0\%).

There were 161 exercise events tracked with the app from 10 participants. The WHRM recorded 116 exercise events from the 10 participants that uploaded data. There were 277 exercise events from both data sources with 80 events that matched in both the app and the WHRM.

\section{Comparison of Exercise Behaviors between the App and WHRM}

The average weekly frequency of exercise was 3.0 (2.1) and 2.5 (1.8) days as tracked with the app and recorded by the WHRM, respectively $(p=0.60)$. On average, participants who self-tracked their exercise with the app recorded an average of 0.3 (1.1) days more of exercise per week than the WHRM. The average duration from 80 matching exercise events from the app was 65.6 (55.2) minutes and from the WHRM was 64.8 (54.9) minutes $(p=0.45)$, with a correlation of 0.90 (-Fig. 2).

Participants self-tracked moderate exercise in 46\% (75/ 161 ) of the cases, with $32 \%$ (51/161) tracked as light, and $22 \%$ (35/161) as vigorous. The average heart rate of the WHRM exercise events were overwhelmingly categorized as moderate in $79 \%(92 / 116)$, with few categorized as vigorous or light, 18\% (21/116) and 3\% (3/116), respectively (-Fig. 3). Participants self-tracked exercise intensity from the app matched the intensity as categorized by the average heart rate from the WHRM in 58\% (46/80) and the highest heart rate in $40 \%(32 / 80)$ of the matched exercise events. When comparing against the average heart rate recorded by the WHRM during exercise, participants on average tracked a lower intensity in $35 \%(28 / 80)$ of exercise and a higher intensity in $7.5 \%$ (6/80). The Cohen's kappa level of concordance for the app against the WHRM average heart rate was 0.2 , a poor to fair level of agreement.

\section{Comparison of $\Delta \mathrm{G} / \mathrm{min}$ between the App and WHRM}

There were 38 of the 277 exercise events for which there were no corresponding CGM readings, resulting in 239 exercise events where $\Delta \mathrm{G} / \mathrm{min}$ could be calculated. The average $\Delta \mathrm{G} / \mathrm{min}$ was greatest for light exercise self-tracked with the app, -0.53 (0.63) $\mathrm{mg} /\left(\mathrm{dL}^{*} \mathrm{~min}\right)$, while the average for vigorous exercise from the app was the least, $-0.19(0.89) \mathrm{mg} /\left(\mathrm{dL}^{*} \mathrm{~min}\right)$. Moderate exercise from the app, light exercise from the WHRM, and moderate exercise from the WHRM were similar, -0.23 (0.99), $-0.29(0.48)$, and $-0.29(0.72) \mathrm{mg} /\left(\mathrm{dL}^{*} \mathrm{~min}\right)$, respectively. Vigorous exercise from the WHRM actually had a slight increase of $0.09(1.0) \mathrm{mg} /$ ( $\mathrm{dL}^{*} \mathrm{~min}$ ). The average $\Delta \mathrm{G} / \mathrm{min}$ was not significantly different based on data source and intensity $(p=0.21)$ and was found to be $-0.27(0.85) \mathrm{mg} /\left(\mathrm{dL}^{*} \mathrm{~min}\right)$ (- Fig. 4).

\section{Discussion}

Measuring exercise behaviors for patients with diabetes is important to researchers, clinicians, and patients. Research studies often rely on exercise sessions for which the variables that influence $\Delta \mathrm{G} / \mathrm{min}$ are highly controlled, while clinical settings often rely on retrospective self-report via questionnaires or surveys to assess physical activity or even more distal outcomes, such as HbA1c scores. Retrospective self-report through surveys and interviews, often the most convenient method for assessment, has shown limited validity and reliability at the individual level for providing insight that can be 

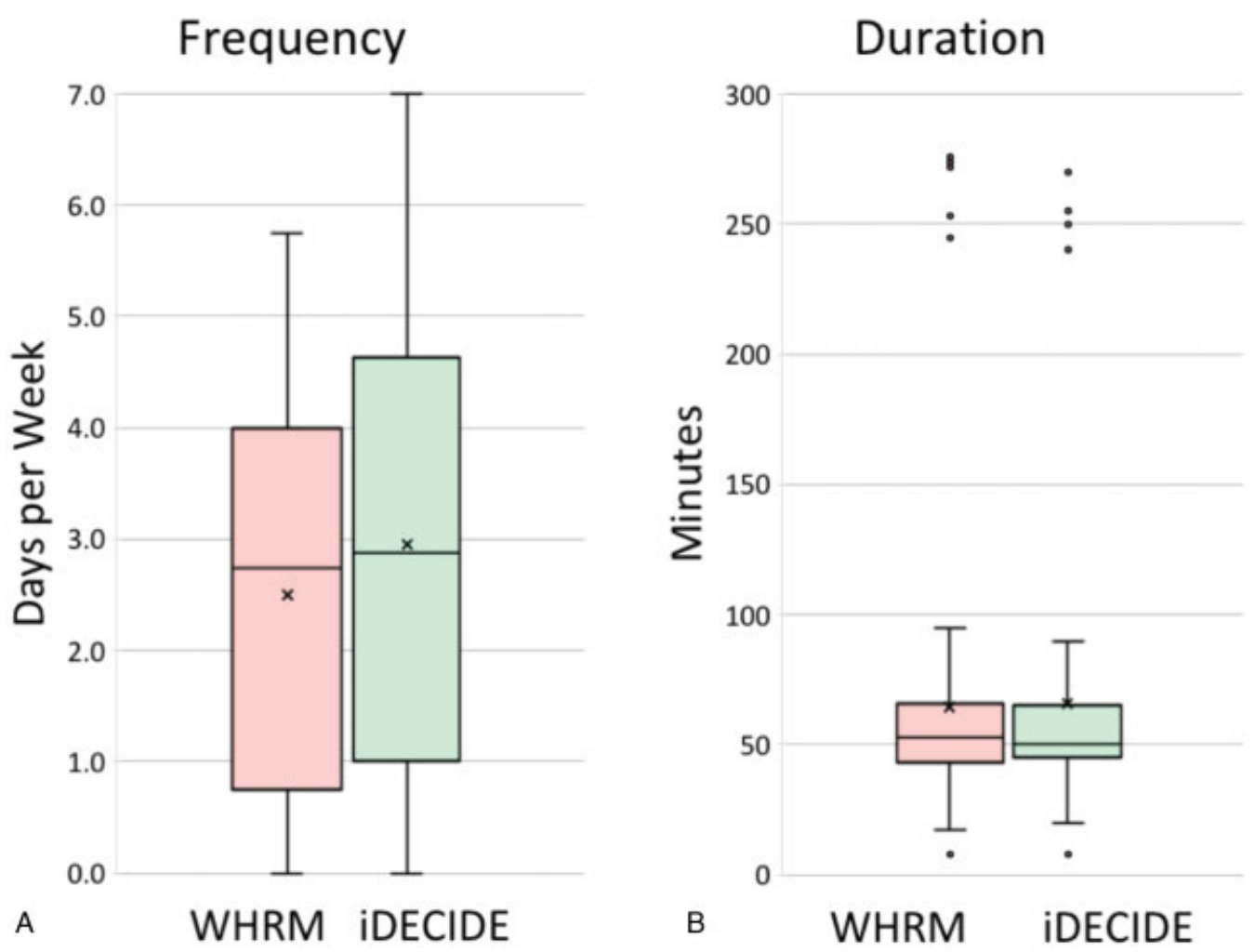

Fig. 2 Exercise self-tracked with the iDECIDE app and recorded with a wristband heart rate monitor (WHRM). There was no significant difference between the two data sources on (A) frequency measured in days per week, and (B) duration measured in minutes.
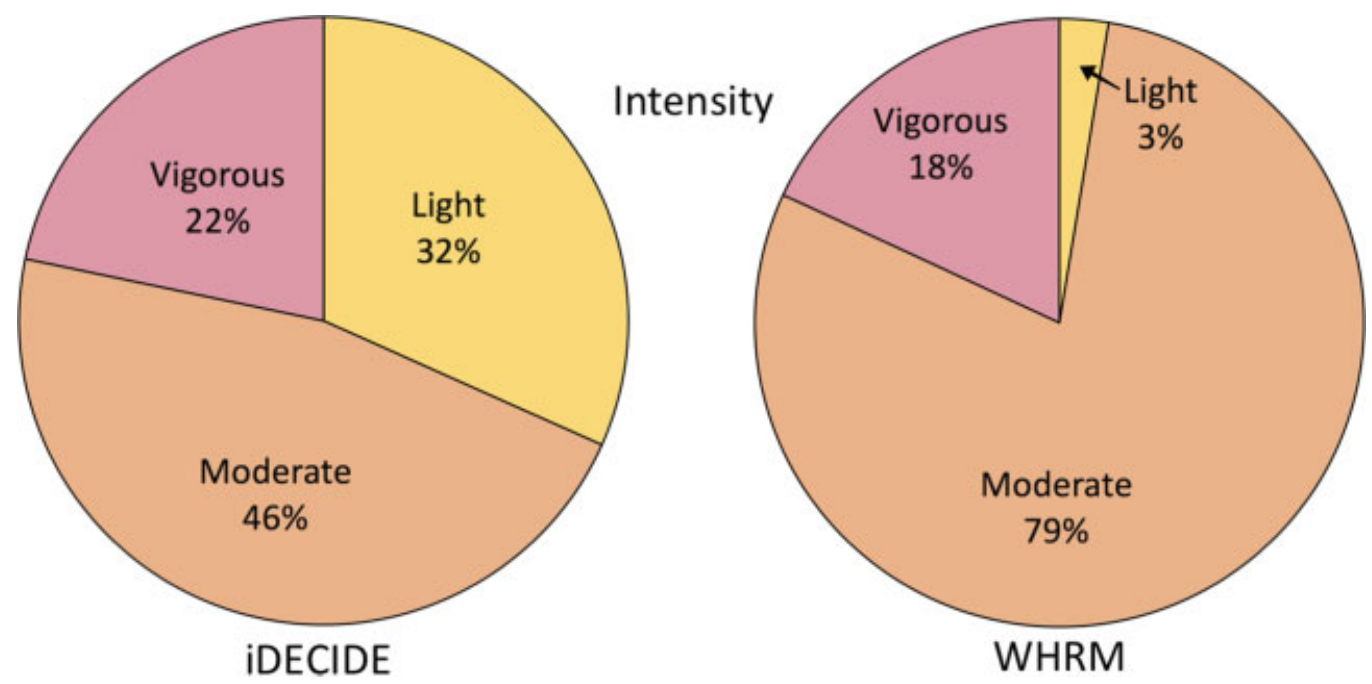

Fig. 3 The pie chart on the left shows the exercise intensity breakdown as self-tracked with the iDECIDE app. The wristband heart rate monitor (WHRM) is based on categorizing intensity from the participant's age and the average heart rate recorded during exercise. The Cohen's kappa level of concordance for self-tracking with the app against the average heart rate from the WHRM was 0.2, a poor to fair level of agreement.

actionable for clinicians. ${ }^{18,30}$ In clinical settings, providers use electronic health records with varying levels of sophistication of clinical decision support for diabetes, with few systems capable of incorporating patient-generated data. ${ }^{31,32}$ Therefore, clinicians are faced with interpreting patient-reported data in the form of paper and digital logs as well as data generated from various devices, such as activity trackers, without any guidance. ${ }^{33}$

Others have noted that technical errors occur with wearables devices, such as unreliable measurements and missing data, which we also experienced in this study. For example, one participant's insulin pump was shifted for 1 hour, while another participant could not upload their WHRM data. Accelerometers, pedometers, multiple sensing systems, global positioning systems, and mathematical models are all methods that can be used to assess physical activity. While each method has its own strengths and weaknesses, for our study we chose to use an off-the-shelf WHRM with proprietary algorithms to gather information on specific exercise events rather than daily totals of activity. The testing of the validity 


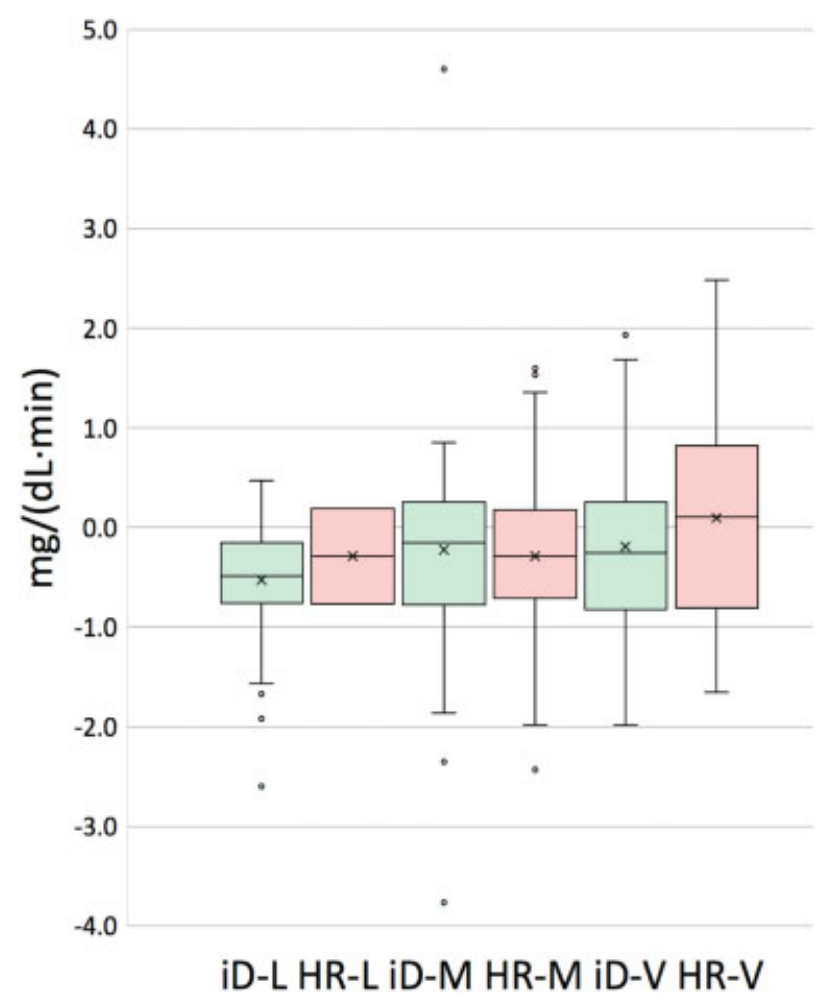

Fig. 4 The rate of change in glucose was calculated for exercise selftracked with the iDECIDE app (iD) and recorded by the wristband heart rate monitor (HR). Exercise intensity was categorized as light (L), moderate $(M)$, or vigorous $(\mathrm{V})$. There was no significant difference in the rate of change of glucose between the intensity as categorized by the app and the HR.

and reliability of wearables is still underway, with commercially available heart rate monitors known to lose accuracy for very low and very high heart rates, but overall having high concordance with the gold standard electrocardiogram..$^{19,34}$ The low- and high-end limitations of WHRM may have influenced the exercise events to primarily be categorized as moderate intensity in our study. Unfortunately, in our study there were technical difficulties that resulted in lost data, and possibly erroneous values due to user entry mistakes and inaccurate device measurements. For example, 80 events aligned between the app and the WHRM. Although this study was not designed to capture the reasons for unmatched data points, there were few cases where the WHRM detected physical activity not tracked by the participants. However, many of the unmatched cases could be due to not wearing, charging, or syncing the WHRM. The discrepancy could also be attributed to participants recording exercise with the app after the event and forgetting to adjust the app's default time, which was the current time on the phone.

Diabetes devices, for example, insulin pumps, glucose meters, and CGM, are capable of storing objectively gathered data. The validity of CGM has been studied, with one recent study demonstrating that sensor accuracy of two commercially available CGM sensors was slightly diminished during physical activity when compared with a resting state. ${ }^{14}$ While we observed $\Delta \mathrm{G} / \mathrm{min}$ of $-0.3 \mathrm{mg} /\left(\mathrm{dL}^{*} \mathrm{~min}\right)$ from the $\mathrm{CGM}$, a meta-analysis of $\Delta \mathrm{G} / \mathrm{min}$ in controlled exercise sessions found that for continuous moderate exercise $\Delta \mathrm{G} / \mathrm{min}$ was $-1.33 \mathrm{mg} /$ ( $\mathrm{dL}^{*} \mathrm{~min}$ ), a fourfold larger impact on $\Delta \mathrm{G} / \mathrm{min}$ than what we observed. ${ }^{35}$ This discrepancy may be due to the controlled situations and differences in devices and methods for measuring glucose. For example, interstitial glucose measures, like those used in our study from the CGM, are known to lag behind blood glucose measurements, the common method used to validate CGM and the gold standard in exercise studies of patients with T1D. ${ }^{14,36}$ It may also be due to the participants not receiving specific instructions to calibrate the CGM and that sensors from different manufacturers were used by the participants, which would introduce noise into the glucose data. Also, participants in this study engaged in compensation techniques to offset the effects of exercise, such as disconnecting from the pump or taking a snack, as previously reported on this study cohort. ${ }^{25}$

Our study is the first to take heterogeneous data from three sources used by patients in clinical settings and compare exercise behaviors to better understand their concordance in free-living patients. We had a small sample size that was mostly female, all of which owned smartphones, which limits the external validity of this study. Unique to this study and prior work by the authors is the focus on adult patients with T1D, as the majority of studies on exercise in free-living patients with T1D are conducted in youth and emerging adults. ${ }^{37-39}$ We found that participants self-tracking aligned well with the WHRM with respect to frequency and duration, which may have been due to the presence of the WHRM making patients more likely to self-track with the app. The intensity of exercise self-tracked had low levels of agreement with the WHRM. The discrepancy in intensity could be due to participants' underreporting intensity, inaccuracies in WHRM due to limitations of the underlying technology to sense and calculate heart rate, and the thresholds chosen against the maximum heart rate could have miscategorized exercise intensity. ${ }^{13,40}$ However, from a glucose management perspective, there was not a significant difference on $\Delta \mathrm{G} / \mathrm{min}$.

One of the limitations of this study and others that have studied patient- and device-generated data is that there are no standard methods for analyzing and reporting the results. Most have focused on reporting overall ratios of desirable and undesirable behaviors related to logging glucose levels, while in this study we measured levels of agreement and presented averages from participants on behaviors related to exercise. $21,41,42$

Our findings also indicate that self-tracking exercise behaviors with an app like iDECIDE, can be used to gather diabetes self-management behaviors and to facilitate decision making for self-management of glucose levels before engaging in exercise. This may be an attractive feature for researchers conducting studies and clinicians that provide care for patients with diabetes as it streamlines the processes for data gathering, assimilation, and analysis. As for patients on intensive insulin therapy who already must incorporate various supplies and devices into their daily regimen, it eliminates the need to incorporate an additional device to 
wear, charge, and sync to interpret the data during and after exercise.

We found that self-tracked data from the app had very good agreement with the WHRM and that the effect on $\Delta \mathrm{G} /$ min was constant across the devices and intensities. We are currently analyzing data that include survey and app data from this cohort and a recently recruited cohort to contrast survey responses about meal, alcohol, and exercise against behaviors self-tracked with the app. Future work includes continued research with the app in free-living settings to further study how best to incorporate patient-reported exercise into bedside tools to help clinicians better treat patients, such as providing data-driven personalized educational interventions.

\section{Conclusion}

We collated and analyzed data from three heterogeneous sources of data found in outpatient settings from free-living participants with T1D to answer clinically relevant questions. Clinicians and researchers should be aware of the various sources of patient-generated data and their levels of agreement when assessing exercise behaviors related to the self-care of T1D. Self-tracking exercise with an app and exercise recorded by a WHRM resulted in good levels of agreement and the same impact on $\Delta \mathrm{G} / \mathrm{min}$. This result suggests that clinicians can rely on patient-reported exercise data when making treatment recommendations and that patients with T1D can make self-care decisions before engaging in physical activity based on their perceived intensity of the ensuing exercise instead of relying on a wearable device to assist with self-management of glucose levels during or after the physical activity.

\section{Clinical Relevance Statement}

iDECIDE, a smartphone app that allows patients to self-track exercise in real time, can reduce the number of devices patients incorporate into their self-care regimen by eliminating the need to use an additional activity monitoring device.

When making recommendations for incorporating exercise into self-management, clinicians can rely on exercise data gathered in real time, either self-reported with an app or recorded by a wearable device.

\section{Protection of Human and Animal Subjects}

This study was reviewed by the Arizona State University and Mayo Clinic Institutional Review Boards.

\section{Funding}

This research was supported by the 2018 ASU-Mayo Research Accelerator Award: Data-Driven BehavioralChange Individualized Interventions to Improve Type 1 Diabetes.

\section{References}

1 Geller AI, Shehab N, Lovegrove MC, et al. National estimates of insulin-related hypoglycemia and errors leading to emergency department visits and hospitalizations. JAMA Intern Med 2014; 174(05):678-686

2 Aiello LP; DCCT/EDIC Research Group. Diabetic retinopathy and other ocular findings in the diabetes control and complications trial/epidemiology of diabetes interventions and complications study. Diabetes Care 2014;37(01):17-23

3 Martin CL, Albers JW, Pop-Busui R; DCCT/EDIC Research Group. Neuropathy and related findings in the diabetes control and complications trial/epidemiology of diabetes interventions and complications study. Diabetes Care 2014;37(01):31-38

4 American Diabetes Association. Standards of medical care in diabetes-2016. Diabetes Care 2016;39(Suppl 1):S23-S25

5 Kourtoglou GI. Insulin therapy and exercise. Diabetes Res Clin Pract 2011;93(Suppl 1):S73-S77

6 Riddell MC, Gallen IW, Smart CE, et al. Exercise management in type 1 diabetes: a consensus statement. Lancet Diabetes Endocrinol 2017;5(05):377-390

7 Glasgow RE, Anderson RM. In diabetes care, moving from compliance to adherence is not enough. Something entirely different is needed. Diabetes Care 1999;22(12):2090-2092

8 Funnell MM, Anderson RM. Empowerment and self-management of diabetes. Clin Diabetes 2004;22(03):123-127

9 Hood KK, Peterson CM, Rohan JM, Drotar D. Association between adherence and glycemic control in pediatric type 1 diabetes: a meta-analysis. Pediatrics 2009;124(06):e1171-e1179

10 Toni S, Reali MF, Barni F, Lenzi L, Festini F. Managing insulin therapy during exercise in type 1 diabetes mellitus. Acta Biomed 2006;77(Suppl 1):34-40

11 Pinsker JE, Kraus A, Gianferante D, et al. Techniques for exercise preparation and management in adults with type 1 diabetes. Can J Diabetes 2016;40(06):503-508

12 Grando MA, Groat D, Soni H, et al. Characterization of exercise and alcohol self-management behaviors of type 1 diabetes patients on insulin pump therapy. J Diabetes Sci Technol 2017;11(02):240-246

13 Sallis JF, Saelens BE. Assessment of physical activity by self-report: status, limitations, and future directions. Res Q Exerc Sport 2000; 71(Suppl 2):1-14

14 Taleb N, Emami A, Suppere C, et al. Comparison of two continuous glucose monitoring systems, Dexcom G4 Platinum and Medtronic Paradigm Veo Enlite System, at rest and during exercise. Diabetes Technol Ther 2016;18(09):561-567

15 Wen D, Zhang X, Liu X, Lei J. Evaluating the consistency of current mainstream wearable devices in health monitoring: a comparison under free-living conditions. J Med Internet Res 2017;19(03):e68

16 Evenson KR, Goto MM, Furberg RD. Systematic review of the validity and reliability of consumer-wearable activity trackers. Int J Behav Nutr Phys Act; December 18, 2015;12. Available at: https://www. ncbi.nlm.nih.gov/pmc/articles/PMC4683756/. Accessed September 3, 2018

17 Deka P, Pozehl B, Norman JF, Khazanchi D. Feasibility of using the Fitbit ${ }^{\circledR}$ Charge HR in validating self-reported exercise diaries in a community setting in patients with heart failure. Eur J Cardiovasc Nurs 2018;17(07):605-611

18 Gonzalez JS, Schneider HE. Methodological issues in the assessment of diabetes treatment adherence. Curr Diab Rep 2011;11 (06):472-479

19 Kozey-Keadle S, Libertine A, Lyden K, Staudenmayer J, Freedson PS. Validation of wearable monitors for assessing sedentary behavior. Med Sci Sports Exerc 2011;43(08):1561-1567

20 Butte NF, Ekelund U, Westerterp KR. Assessing physical activity using wearable monitors: measures of physical activity. Med Sci Sports Exerc 2012;44(01, Suppl 1):S5-S12

21 Blackwell M, Wheeler BJ. Clinical review: the misreporting of logbook, download, and verbal self-measured blood glucose in 
adults and children with type I diabetes. Acta Diabetol 2017;54 (01):1-8

22 Trawley S, Baptista S, Browne JL, Pouwer F, Speight J. The use of mobile applications among adults with type 1 and type 2 diabetes: results from the Second MILES-Australia (MILES-2) Study. Diabetes Technol Ther 2017;19(12):730-738

23 Dobson R, Whittaker R, Murphy R, et al. The use of mobile health to deliver self-management support to young people with type 1 diabetes: a cross-sectional survey. JMIR Diabetes 2017;2(01):e4

24 Lithgow K, Edwards A, Rabi D. Smartphone app use for diabetes management: evaluating patient perspectives. JMIR Diabetes 2017;2(01):e2

25 Groat D, Soni H, Grando MA, Thompson B, Kaufman D, Cook CB. Design and testing of a smartphone application for real-time selftracking diabetes self-management behaviors. Appl Clin Inform 2018;9(02):440-449

26 Centers for Disease Control and Prevention. Measuring Physical Activity Intensity. CDC 24/7: Saving Lives, Protecting People; 2015. Available at: https://www.cdc.gov/physicalactivity/basics/ measuring/index.html. Accessed June 7, 2018

27 Reed JL, Pipe AL. The talk test: a useful tool for prescribing and monitoring exercise intensity. Curr Opin Cardiol 2014;29(05): 475-480

28 FitBit. Charge HR 101. Available at: https://www.fitbit.com/c/ chargehr/chargehr-101. Accessed June 17, 2017

29 Tanaka H, Monahan KD, Seals DR. Age-predicted maximal heart rate revisited. J Am Coll Cardiol 2001;37(01):153-156

30 Sylvia LG, Bernstein EE, Hubbard JL, Keating L, Anderson EJ. Practical guide to measuring physical activity. J Acad Nutr Diet 2014;114(02):199-208

31 Kantor M, Wright A, Burton M, et al. Comparison of computerbased clinical decision support systems and content for diabetes mellitus. Appl Clin Inform 2011;2(03):284-303

32 Kumar RB, Goren ND, Stark DE, Wall DP, Longhurst CA. Automated integration of continuous glucose monitor data in the electronic health record using consumer technology. J Am Med Inform Assoc 2016;23(03):532-537
33 O'Connor P. Opportunities to increase the effectiveness of EHRbased diabetes clinical decision support. Appl Clin Inform 2011;2 (03):350-354

34 Wang R, Blackburn G, Desai M, et al. Accuracy of wrist-worn heart rate monitors. JAMA Cardiol 2017;2(01):104-106

35 García-García F, Kumareswaran K, Hovorka R, Hernando ME. Quantifying the acute changes in glucose with exercise in type 1 diabetes: a systematic review and meta-analysis. Sports Med 2015;45(04):587-599

36 Matuleviciene V, Joseph JI, Andelin M, et al. A clinical trial of the accuracy and treatment experience of the Dexcom G4 sensor (Dexcom G4 system) and Enlite sensor (guardian REAL-time system) tested simultaneously in ambulatory patients with type 1 diabetes. Diabetes Technol Ther 2014;16(11):759-767

37 Hendricks M, Monaghan M, Soutor S, Chen R, Holmes CS. A profile of self-care behaviors in emerging adults with type 1 diabetes. Diabetes Educ 2013;39(02):195-203

38 Pyatak EA, Carandang K, Vigen C, et al. Resilient, Empowered, Active Living with Diabetes (REAL Diabetes) study: methodology and baseline characteristics of a randomized controlled trial evaluating an occupation-based diabetes management intervention for young adults. Contemp Clin Trials 2017;54:8-17

39 Yardley JE, Hay J, Abou-Setta AM, Marks SD, McGavock J. A systematic review and meta-analysis of exercise interventions in adults with type 1 diabetes. Diabetes Res Clin Pract 2014;106 (03):393-400

40 Schäfer A, Vagedes J. How accurate is pulse rate variability as an estimate of heart rate variability? A review on studies comparing photoplethysmographic technology with an electrocardiogram. Int J Cardiol 2013;166(01):15-29

41 Mazze RS, Shamoon H, Pasmantier R, et al. Reliability of blood glucose monitoring by patients with diabetes mellitus. Am J Med 1984;77(02):211-217

42 Guilfoyle SM, Crimmins NA, Hood KK. Blood glucose monitoring and glycemic control in adolescents with type 1 diabetes: meter downloads versus self-report. Pediatr Diabetes 2011;12(06): 560-566 